\title{
R\&D in the Pharmaceutical Industry: A World of Small Innovations ${ }^{1}$
}

\author{
Beatriz Domínguez ${ }^{2} \quad$ Juan José Ganuza ${ }^{3} \quad$ Gerard Llobet ${ }^{4}$
}

December, 2005

\footnotetext{
${ }^{1}$ We thank the audience of the Health Economics Seminar at the Universidad Carlos III for useful comments. All redundancies are due to the second author. All missing explanations are due to the third one. Otherwise, the usual disclaimer applies.

${ }^{2}$ BBVA.

${ }^{3}$ Department of Economics, Universitat Pompeu Fabra. C/ Ramon Trias Fargas 25-27 08005 Barcelona, Spain. E-mail: juanjo.ganuza@econ.upf .es.

${ }^{4}$ CEMFI. C/ Casado del Alisal 528014 Spain. E-mail:1lobet@cemfi.es.
} 


\begin{abstract}
It is commonly argued that in recent years pharmaceutical companies have directed their R\&D towards small improvements of existing compounds instead of more risky drastic innovations. In this paper we show that the proliferation of these small innovations is likely to be linked to the lack of market sensitivity of a part of the demand to changes in prices. Compared to their social contribution, small innovations are relatively more profitable than large ones because they are targeted to the smaller but more inelastic part of the demand. We also study the effect of regulatory instruments such as price ceilings, copayments and reference prices and extend the analysis to competition in research.
\end{abstract}

JEL Codes: I11, I18, L51, O31.

Keywords: health-care, pharmaceuticals, innovation. 


\section{Introduction}

The pharmaceutical industry channels an important proportion of total research in most developed countries. In Europe, for example, it represents around the $13 \%$ of total R\&D. In recent years pharmaceutical companies have been accused of devoting their resources mainly to minor improvements over existing medications that require short clinical trials and have a small risk of not being approved. In a survey for the U.S. by the National Institute for Health Care Management (NIHCM (2002)) it is reported that only $17 \%$ of all the drugs approved by the FDA in the period 1989-2000 can be classified as drastic innovations, while $51 \%$ were mere modifications of existing compounds. ${ }^{1}$ At the same time, it has been documented that pharmaceutical companies have increased substantially their investment in advertising and marketing of their products. According to some estimates, advertising expenditures in the U.S. multiplied by a factor of three between 1998 and $2004 .^{2}$

In this paper, we show that the recent trend in the pharmaceutical industry towards small innovations is related to the low sensitivity of the demand. We show that the rewards that innovators obtain are distorted in a systematic way with respect to the social contribution they provide. In particular, small innovations get a proportionally larger reward because pharmaceutical firms target them to the inelastic part of the doctor's induced demand. This lack of elasticity is due to the subsidy by public health agencies and HMOs of the cost of these medications. As a consequence, firms find relatively more profitable to invest in small innovations. Advertising contributes to this strategy by attracting inelastic buyers that are otherwise insensitive to price changes.

Small innovations are particularly visible in two contexts. They might be the response to the entry of generic products or the result of competition with products patented by competitors. In the first case, many countries grant an extension of the monopoly power of firms that provide improvements (albeit minor) of their products, for which a new product patent would not be granted. In the U.S., for example, the Hatch-Waxman Act (1984) grants an extension

\footnotetext{
${ }^{1}$ See Lexchin (2003) and Love (2003) for additional evidence on the bias toward small innovations.

${ }^{2}$ See The Economist, March 17th 2005.
} 
of three years for "incrementally modified drugs" (IMD) and five years for "new molecular entities" (NME). ${ }^{3}$ Moreover, in some countries modifications of existing compounds are entitled to process patents that extend the innovator's protection after the expiration of the original patent.

In the second case, pharmaceutical firms often engage in the modification of successful products marketed by competitors in expanding markets as a way to steal profits. These are often denoted me-too drugs. The market for statins is a case in point. Statins are cholesterol-lowering drugs that appeared in the 1990s. Starting from Lovastatin, several firms have introduced competing varieties of the compound like simvastatin (Zocor), atorvastatin (Lipitor), pravastatin (pravachol), fluvastatin (Lescol) or rosuvastatin (Crestor). These products are claimed to be close substitutes, and arguably they involve a lower risk and lower investment than the development of more innovative products. ${ }^{4}$

We aim to understand why firms in this market tend to target their research to these small improvements. Starting with Nordhaus (1969), existing literature on innovation has commonly argued that to the extent that firms do not internalize all the surplus of the innovations they generate, underinvestment is likely to arise. The existence of patents is seen as a way to address this inefficiency and classical papers such as Gilbert and Shapiro (1990) and Klemperer (1990) have studied the trade-offs of longer versus wider patents. Recent papers, such as Scotchmer (1999), have shown that patents are also efficient tools to relate the value of the invention to the social reward it generates. In this paper, we argue that in obtaining the optimal level of innovation an additional margin is important. As the previous examples illustrate, firms typically choose the size of the innovation they pursue, and for this reason, underinvestment (or overinvestment) will be a function of how close is the contribution for each size of innovation they might achieve to the social welfare they generate. Misallocation of resources will occur in markets where the signals originating from the demand for the good are dampened in a systematic way.

\footnotetext{
${ }^{3}$ See Bulow (2004) for a review of the Hatch-Waxman Act, and the effects on patentholders and potential generic producers.

${ }^{4}$ See Hollis (2005) for a more in-depth discussion of these issues.
} 
The results we obtain are related to the particular structure of the demand for pharmaceutical products, compared to other markets where innovation is important. Patients often do not pay nor they choose the medications they purchase, as they are prescribed by their physicians. For this reason, how much the demand is price sensitive depends on how much physicians internalize the real cost of the medication as opposed to internalizing the preferences of their patients. In the second case, they might align with their patients if for example they are concerned with maximizing the success rate of a treatment at any cost or if they believe that the prescription of a heavily advertised branded product instead of a generic one affects the patient's perception of the treatment. ${ }^{5}$ Hellerstein (1998) finds indeed empirical evidence of this differentiated behavior. As Scherer (1993) states

"it is not too extreme an oversimplification to suppose that when generic substitutes exist, the world of drug buyers consists of two quite different groups - those that are price-sensitive and those who are not. When there is only one branded product, the pricing problem is straightforward. But once generic substitutes enter at much lower prices, the market is bifurcated, and the incumbent branded seller commonly finds it more profitable to desert the price-sensitive market than to reduce the prices quoted to price-sensitive customers."

Along those lines, the model we construct includes two kinds of induced demand. Those doctors that internalize the preferences of their patients are denoted as captured doctors. The rest of the doctors, that take into account the benefit as well as the cost of the several medications, are denoted as non-captured doctors. ${ }^{6}$ Pharmaceutical firms might choose between setting a high price in order to sell to the captured part of the demand or to charge a rather lower price in order to also attract the rest of the patients. For firms with small innovations,

\footnotetext{
${ }^{5}$ Other alternative explanations for the low demand elasticity are related to the lack of incentives of doctors to be informed about the quality of products and their price. See for example Caves et al. (1991) and Danzon and Chao (2000).

${ }^{6}$ Along similar lines Frank and Salkever (1992) propose a model where the demand for a patented product is divided into two segments depending on whether they are sensitive to the price of the generic product or not. However, they are not concerned with the incentives for further research to be undertaken.
} 
a higher price compensates for the lower level of sales, while for big improvements, serving the whole market is optimal, since the willingness to pay of price-sensitive consumers is higher.

Compared to the social optimum, the lack of price-sensitivity of the demand provides an excessive reward for small innovations and consequently it distorts the incentives of pharmaceutical firms. As a result, firms underinvest in R\&D. As mentioned earlier, this result does not arise from the lack of rents from innovation but rather on how these rents are distributed across different possible improvements.

To address the excessive market power of pharmaceutical firms and reduce the medical expenditures, additional regulations are in place in most countries. Although they were not intended to modify the incentives of firms to innovate they have clear effects. As an example, we study the likely effects on the resulting innovation of three commonly used instruments: price ceilings, copayments and reference prices. ${ }^{7}$

Pharmaceutical firms often argue that this kind of price controls, to the extent that they reduce the profits from innovation, lead to a lower level of research. The lesson from our study is that although these instruments might help reduce excessive rents derived from the distorted demand, they might also provide more incentives (sometimes even excessive) to undertake research. The result depends on how each instrument affects the resulting demand elasticity. In this respect, there is a clear trade-off between the kind of insurance that creates the distorted demand in the first place and the possibility to align the incentives of innovators and society.

Price ceilings, for example, still insure perfectly the patient's expenses, while at the same time they limit the monopoly power of the pharmaceutical firm. Trivially, if the insurer had perfect information regarding the social contribution of the improvement, a price ceiling contingent on the characteristics of the innovation would implement the first best. To the extent that this is not possible, and this is one of the reasons why patents exist, a price ceiling does not help to make the demand more elastic to changes in the price. As a result, large innovations might receive smaller than optimal rewards and induce even lower investment.

\footnotetext{
${ }^{7}$ Although we analyze them separately, in practice they often co-exist. For example, in Germany, patients typically pay $10 \%$ of the price with a minimum of 5 euros and a maximum of 10 euros. They also cover the excess price difference using reference pricing.
} 
Copayments and reference prices affect directly the payment that patients make of their prescriptions. Consequently, they reduce the insurance provided but lead to a more elastic demand. However, because copayments subsidize a percentage of the cost of the prescription while reference prices subsidize a constant amount the results are different. Reference prices only induce the first best when no insurance exists, while this outcome can be achieved with a more modest copayment.

The last section of the paper generalizes the model to the case of competition in innovation among pharmaceutical firms. We devise an environment in which under competition the first best can be achieved if the demand is perfectly sensitive to the price. Captured doctors have in this case an ambiguous effect on the level of innovation due to two opposing forces. On the one hand, a less sensitive demand provides more incentives to each firm to reach an improvement that supercedes the innovation that the rival achieves. On the other, contingent on superseding the rival, the demand becomes very little sensitive to further improvements. We show by means of example that the second force is likely to dominate when the risk of the R\&D process is large, leading to underinvestment.

The paper proceeds as follows. The next section introduces the benchmark model. Section 3 discusses the effects of each of the instruments and section 4 analyzes the scenario with competition. Section 5 concludes.

\section{The Model}

Consider a market where a number of firms is producing an existing good. We normalize its quality to 0 . One of the firms, firm $i$, can invest in increasing its quality by performing R\&D. Firm $i$ makes effort $\theta$ to obtain a good of random quality $v$ in the interval $[0,1]$. The quality of the good originates from the distribution $F(v, \theta)$ with density $f(v, \theta)$. We assume that the distribution $F$ is ordered in the first-order stochastic sense, so that more effort increases the probability of obtaining a good of high quality; if $\theta^{\prime}>\theta$ then $F\left(v, \theta^{\prime}\right)<F(v, \theta)$ for all $v$. The 
cost of performing effort $\theta$ is also denoted as $\theta .^{8}$

We assume that the marginal cost of production of all goods is normalized to 0 . As a result of competition, the original good has an equilibrium price of 0 , while firm $i$ sets a positive price $p$ for its improved good. ${ }^{9}$

The demand side of the market corresponds to a unit mass of patients with a completely inelastic demand for a single unit of the good. Each patient derives utility $U(v)=v$. We initially assume that patients do not pay directly for the good, and the cost is incurred instead by an insurance company. We further assume that regulation sets a price ceiling, which is limited to 1.

The demand for the good is induced by the doctors that prescribe the medication. There are two kinds of doctors. A proportion $\sigma$ are denoted as captured doctors (C), that prescribe the good with the highest quality regardless of its price. The remaining proportion $1-\sigma$ of noncaptured doctors (NC) assign the medication according to a cost-efficiency analysis. Captured doctors' preferences can be represented by a utility function

$$
U_{C}(v)=U(v)=v
$$

which can be interpreted as collusion between doctors and their patients. Non-captured doctors take into account the valuation as well as the price of the good according to the utility function

$$
U_{N C}(v, p)=v-p
$$

so that they choose as if patients themselves had to pay the price of the medication. In other words, they consider the total cost and benefit for the patient and the insurer.

The timing of the game is as follows. In the first stage, firm $i$ chooses effort $\theta$. In the second stage the quality $v$ is realized and market competition leads to equilibrium prices and allocation of the goods.

\footnotetext{
${ }^{8}$ By redefining the density function $f$, any cost function $C(\theta)$ would lead to the same results in as long as $C$ is increasing in $\theta$.

${ }^{9}$ For this equilibrium to exist we only require that at least one firm other than firm $i$ produces the original good. This is consistent with the two kinds of examples discussed in the introduction. That is, the original market could be served with generic products after the expiration of a patent, possibly of firm $i$. Alternatively, the improvement of firm $i$ does not infringe the patent owned by the creator of the existing product.
} 


\subsection{The First Best}

From the social point of view, the optimal allocation of the goods is simple. Given that the marginal cost of production is 0 and all patients are alike, they ought to consume the good with quality $v$. Hence, the social value that an innovation of size $v$ generates is precisely $S(v)=v$, which means that the optimal level of effort can be defined as,

$$
\theta^{S} \in \arg \max _{\theta} \int_{0}^{1} v f(v, \theta) d v-\theta
$$

To avoid trivial results, we focus on the case in which the socially optimal level of investment is positive.

\subsection{The Market Equilibrium}

In the private solution, the price $p$ that firm $i$ sets in the second stage, and the ensuing profits, affect the innovative effort that the firm chooses. In spite of the assumption that patients do not pay for the price of the good, the demand of firm $i$ will depend on the price because some doctors are price sensitive when prescribing the good. These doctors only choose the good sold by firm $i$ when it provides net value $v-p$ larger than what the homogeneous product would provide, 0 .

Hence, for a given quality $v$, firm $i$ might essentially maximize profits by choosing two different prices depending on whether only the captured doctors prescribe the drug or all doctors prescribe it. For the first, the maximum price allowed, $p=1$ is optimal, resulting in sales of $\sigma$. For the second, the optimal price is defined as $p=v$ so that all doctors prescribe the drug and sales are 1. By comparing the profits from both options we can derive the following lemma.

Lemma 1 For high quality improvements, $v \geq \sigma$ the optimal price is $p=v$ so that firm $i$ sells to all patients. Otherwise, the optimal price is $p=1$ and a proportion $\sigma$ of patients buys the improved product. 
Interestingly, while this model naturally predicts that larger innovations are associated with higher profits, they do not necessarily lead to a higher price for the good.

In the first stage, the effort decision of firm $i$ depends on the profits obtained from the sale of the good. In particular, because profits from a small and a large improvement correspond to $\sigma$ and $v$ respectively, the privately optimal effort decision of firm $i$ can be characterized by the expression

$$
\theta^{*}(\sigma) \in \arg \max _{\theta} \int_{0}^{\sigma} \sigma f(v, \theta) d v+\int_{\sigma}^{1} v f(v, \theta) d v-\theta .
$$

Notice that for $\sigma=0$ the private choice of effort coincides with the first best, since the innovator captures all the surplus generated from the production of the good and $\theta^{*}=\theta^{S}$. However, for larger values of $\sigma$ distortions will in general arise. In particular, a higher proportion of captured doctors implies that the profits of firm $i$ for a larger range of low realizations of $v$ are higher than the social value they generate. This is so, because the improvement generates a social value of $v$, yet the firm obtains profits $\sigma>v$. As Figure 1 shows, this distortion reduces the incentives to exert innovative effort because low realizations of $v$ are to some extent insured by the inelastic preferences of captured doctors. We summarize these result in the next proposition.

Proposition 1 The private optimal choice of effort is lower than the first best for all $\sigma>0$. Moreover, the level of effort $\theta^{*}(\sigma)$ is decreasing in $\sigma$.

Notice that, contrary to other models of innovation, lower values of $\sigma$ are associated with lower expected profits from innovation yet they provide more incentives to invest.

The underinvestment result is related to the low elasticity of the demand rather than the particular construction we have used. In Appendix $A$ we develop a model that delivers the same results and assumes away the existence of a price ceiling and considers the dead-weight loss from the market power that the pharmaceutical firm might exercise. As this extension points out, underinvestment occurs as long as the profit (which we denote as $\pi(v)$ ) from a larger innovation increases less than the social contribution it creates, $\pi^{\prime}(v)<1$. 


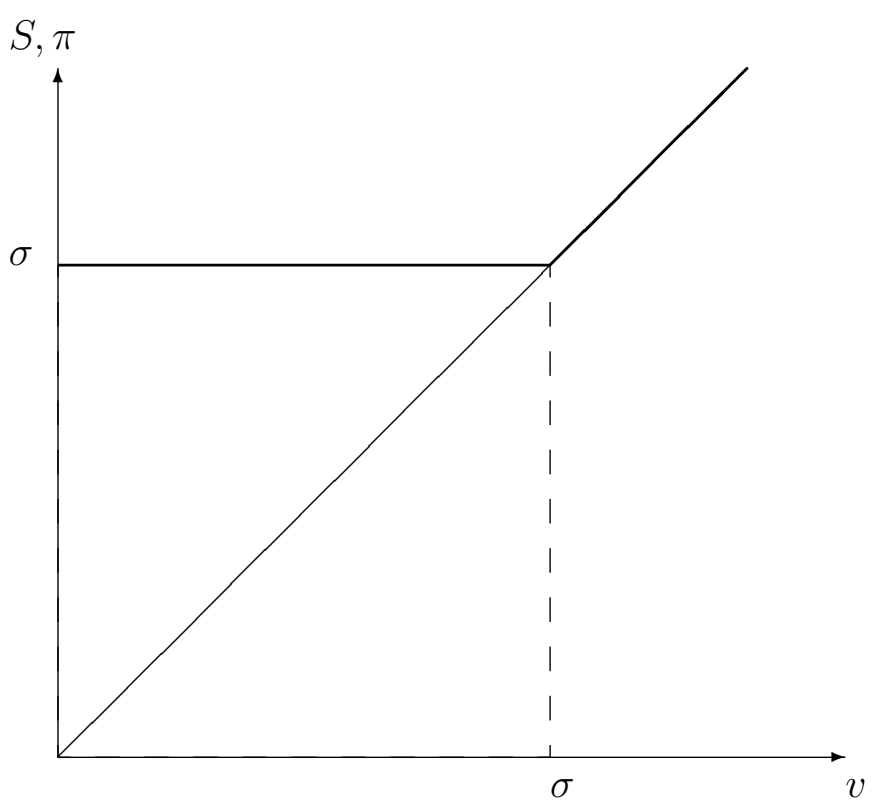

Figure 1: As in the following figures, for different realizations of $v$, the thin diagonal denotes social value $S$ while profits corresponds to the thick line.

\section{Impact of the Regulatory Instruments on R\&D}

In the previous section we have studied the R\&D decision of firms under a distorted demand resulting from the doctors' decisions. Insurance companies and public health agencies react to the resulting inelasticity of the demand by introducing complex regulatory instruments aimed to align their own goals and the incentives of the doctors, towards the rationalization of the expenditure on pharmaceutical products.

In this section we provide an overview of the likely effects of the main regulatory instruments over the incentives of firms to engage in $R \& D$. To the extent that regulation is local but firms innovate for a global market the analysis we present does not have normative content. It does not intent to characterize the optimal policy but rather to outline the effectiveness of these instruments.

The three classical instruments we analyze are price ceilings, copayments and reference prices. Although each instrument has different consequences our analysis conveys the message 
that regulations that increase the elasticity of the demand have in general a positive effect on innovation. Hence, contrary to what pharmaceutical firms often claim, regulations that reduce their rents might still induce more research.

\subsection{The Price Ceiling}

In the benchmark case, we have normalized the maximum price that the firm can charge (the price ceiling) to 1. Part of the distortions we have identified originate from the excessive rents that this price ceiling provides to the firm. An obvious remark, therefore, is that if the regulator has full information about the value of the innovation, a price ceiling of $v$ trivially implements the first best. However, under imperfect information one may wonder whether decreases in the price ceiling have positive effects on the investment of firm $i$. As we now show the results are in general ambiguous.

Given a price ceiling $\tilde{p}$, the characterization of the optimal price of firm $i$ is similar to the benchmark case. Only two prices might maximize profits for the firm. If the firm targets only captured doctors, it chooses a price $\tilde{p}$ that yields profits $\pi=\sigma \tilde{p}$. When the firm targets all patients, a price of $v$ is chosen as long as $v<\tilde{p}$ and $p=\tilde{p}$ otherwise. Profits correspond to $\pi=p$. The next lemma summarizes these results.

Lemma 2 The profit maximizing price corresponds to

$$
p= \begin{cases}\tilde{p} & \text { if } v \geq \tilde{p} \\ v & \text { if } \sigma \tilde{p}<v<\tilde{p} \\ \tilde{p} & \text { if } v \leq \sigma \tilde{p}\end{cases}
$$

Figure 2 shows that the price ceiling does not only apply to small innovations but also has effects on large innovations, the price of which is bounded by $\tilde{p}$. This additional distortion has important effects on the choice of investment of firm $i, \theta_{P C}^{*}$. In particular, the optimal investment level corresponds to

$$
\theta_{P C}^{*} \in \arg \max _{\theta} \int_{0}^{\sigma \tilde{p}} \sigma \tilde{p} f(v, \theta) d v+\int_{\sigma \tilde{p}}^{\tilde{p}} v f(v, \theta) d v+\int_{\tilde{p}}^{1} \tilde{p} f(v, \theta) d v-\theta .
$$




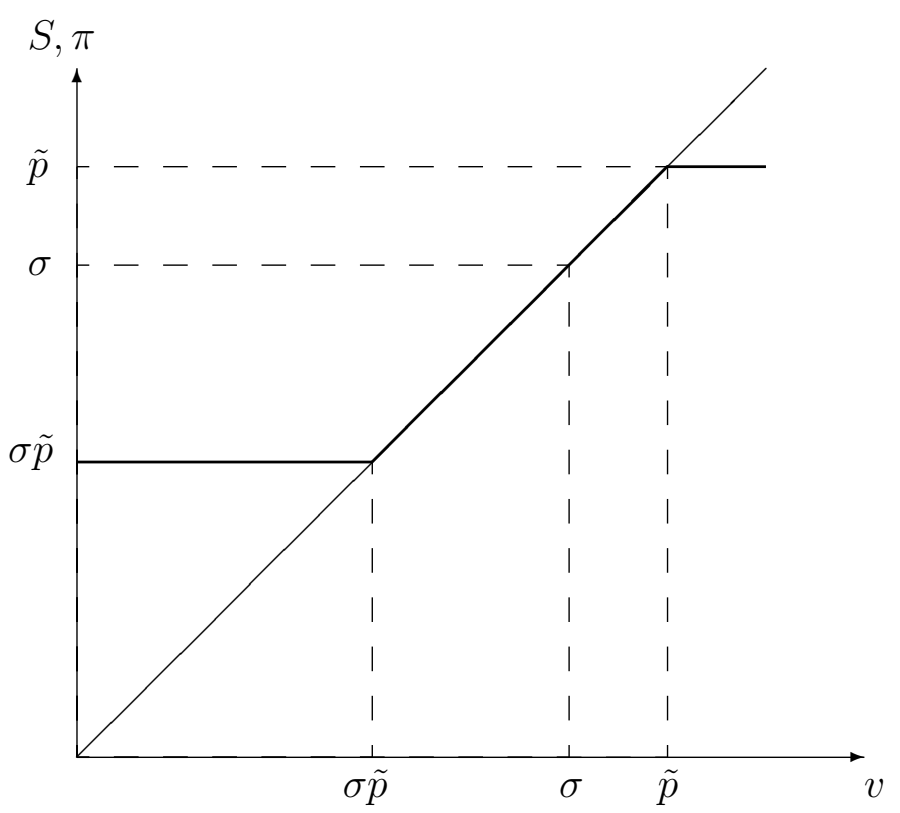

Figure 2: Profits given a reference price $\tilde{p}$.

As opposed to the other instruments we consider next, the effect of a reduction in the price ceiling is in general ambiguous. A lower price ceiling reduces the excessive rents of the firm when low realizations of $v$ occur, enticing it to increase the investment level. But to the extent that a lower price ceiling also reduces the reward when a large innovation is achieved, an opposing force arises. Hence, the direction of the results in general depends on the functional assumptions on the distribution of innovations $F$.

An important implication of the previous remarks is that the first best cannot be in general achieved. A crucial difference between this instrument and copayments or reference prices is that with a price ceiling the patient is still completely subsidized. The other mechanisms operate, instead, by changing the demand elasticity. Given that the reward of the firm can only be aligned with its social contribution through the price sensitiveness of the demand, under full subsidization efficiency is not attainable. 


\subsection{Copayments}

By a copayment we mean a regulatory instrument for which patients pay a fixed percentage $\alpha<1$ of the cost of their prescriptions. The remaining part of the payment, as before, is assumed to be paid by the insurer. Thus, patient preferences are now price-sensitive and can be described according to

$$
U(v, \alpha, p)=v-\alpha p
$$

In the same spirit as we have modelled doctors' preferences in the previous section, we still assume that non-captured doctors, concerned about the cost-efficiency of each medication, do not change their utility function in this case (or in the case of reference prices). Captured doctors, concerned only about the utility of their patients, have their same preferences $U_{C}(v, \alpha, p)=U(v, \alpha, p)$.

The equilibrium prices have features similar to the prices studied in the previous section. Firm $i$ can focus on either the proportion $\sigma$ of captured doctors that are now price-sensitive and are willing to pay a maximum price of $\min \left\{\frac{v}{\alpha}, 1\right\}$ or cover the whole market and let the elastic part of the demand function determine the price. In this last case, the price cannot exceed the value of the improvement, $v$, that the product generates. Hence, firm $i$ targets the inelastic segment of the demand, whenever $\sigma \min \left\{\frac{v}{\alpha}, 1\right\}$ is larger than $v$, or in other words, when the ratio between the proportion of captured doctors and the copayment is large enough. The next lemma characterizes the prices and profits for firm $i$.

Lemma 3 We can distinguish two cases: (i) If $\alpha \leq \sigma$, the equilibrium price is

$$
p= \begin{cases}v & \text { if } v \geq \sigma \\ 1 & \text { if } \alpha<v<\sigma \\ \frac{v}{\alpha} & \text { if } v \leq \alpha\end{cases}
$$

(ii) If $\alpha>\sigma$ then $p=v$.

Profits are $\pi=v$ when $p=v$ and the whole market is covered, and $\pi=\sigma p$ otherwise.

We now turn to the R\&D decision of firm $i$. Denote as $\theta_{C O}^{*}(\sigma, \alpha)$ the investment of the firm when the copayment is set to $\alpha$. In case (ii) of the previous lemma it is clear that since the 


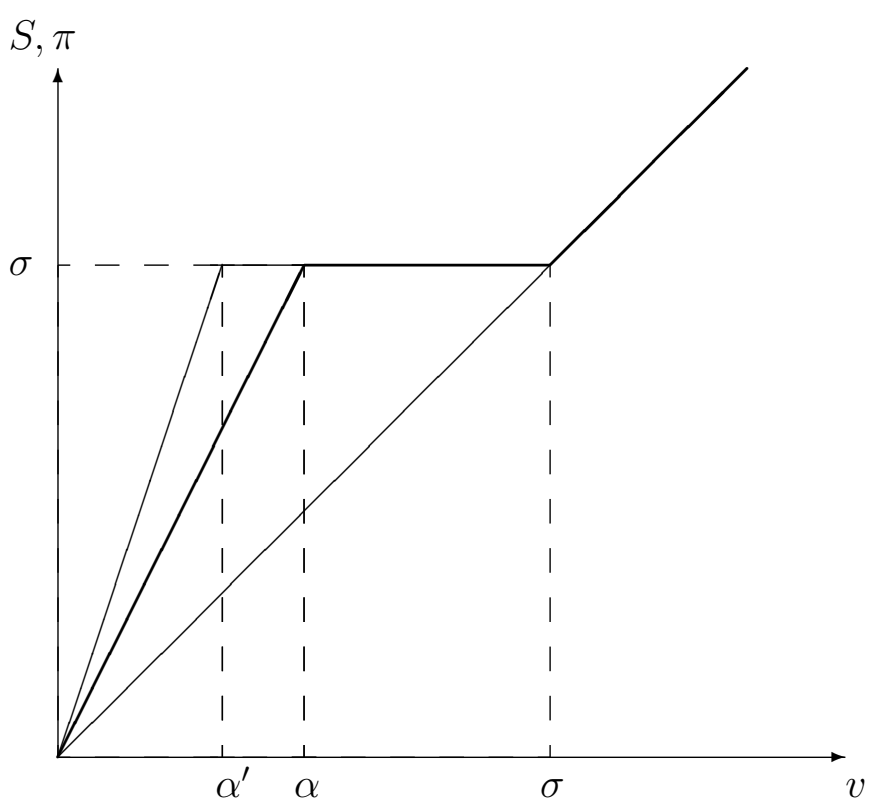

Figure 3: Profits with two levels of copayment $\alpha^{\prime}<\alpha<\sigma$.

price equates the value of the innovation, the incentives of the firm are correctly aligned with the social benefit. Hence, no distortion arises and the first best investment level is achieved, $\theta_{C O}^{*}(\sigma, \alpha)=\theta^{S}$.

In case (i) the level of innovation corresponds to the solution to

$$
\theta_{C O}^{*}(\sigma, \alpha) \in \arg \max _{\theta} \int_{0}^{\alpha} \sigma \frac{v}{\alpha} f(v, \theta) d v+\int_{\alpha}^{\sigma} \sigma f(v, \theta) d v+\int_{\sigma}^{1} v f(v, \theta) d v-\theta .
$$

As Figure 3 illustrates, for low values of $v$ the private profits from the innovation still exceed the social value they generate. However, because patients internalize part of the cost, a positive $\alpha$ means a more elastic demand and a reduction of the rents from small innovations. This intuition gives rise to the following proposition

Proposition 2 For $\alpha \geq \sigma, \theta_{C O}(\sigma, \alpha)=\theta^{S}$. Otherwise, $\theta^{*}(\sigma) \leq \theta_{C O}^{*}(\sigma, \alpha)$.

One of the contributions of this paper is to highlight that, contrary to common wisdom, more rents are not necessarily associated with higher incentives to innovate. In this case, introducing 
a copayment provides more incentives to invest in order to create large improvements, despite the smaller rents that the firm can appropriate.

The previous result does not imply that a higher copayment generates more incentives to invest in R\&D. In fact, the comparative statics are in general ambiguous. To see it, consider an increase in the copayment from $\alpha^{\prime}$ to $\alpha$ as depicted in Figure 3. Such an increase has two differentiated effects. It decreases the marginal return to innovations with $v<\alpha^{\prime}$ but it increases the marginal return of innovations with quality between $\alpha^{\prime}$ and $\alpha$. If a realization of $v$ in the first range is relatively more likely than in the second, a higher copayment might reduce the incentives to invest.

\subsection{Reference Prices}

A reference price is another instrument to regulate pharmaceutical expenditures. It corresponds to a maximum price that the insurer refunds the patient for the prescription. If the price exceeds this reference price, the difference between the actual price and the reference price is borne by the patient. Let $\bar{p}$ be the reference price. Given a price $p$, the utility of the patient can be written as

$$
U(v, p, \bar{p})= \begin{cases}v & \text { if } p \leq \bar{p} \\ v-(p-\bar{p}) & \text { if } p>\bar{p}\end{cases}
$$

Similarly to the previous cases, preferences of non-captured doctors are unchanged, while captured doctors adopt again the preferences of their patients.

As explained earlier, firm $i$ needs to choose whether to only sell to the captured part of the demand or to all patients. In the first situation, the price is set according to the minimum of the price ceiling 1 and the maximum willingness to pay of the patients (of captured doctors), $v+\bar{p}$. In order to sell to all patients, firm $i$ optimally sets $p=v$. Profits in the first case are $\sigma \min \{v+\bar{p}, 1\}$ while in the second, profits are equal to $v$. Hence, larger $\sigma$ and $\bar{p}$ make the equilibrium where the firm serves only a part of the market more likely. The next lemma characterizes the equilibrium prices and profits. 

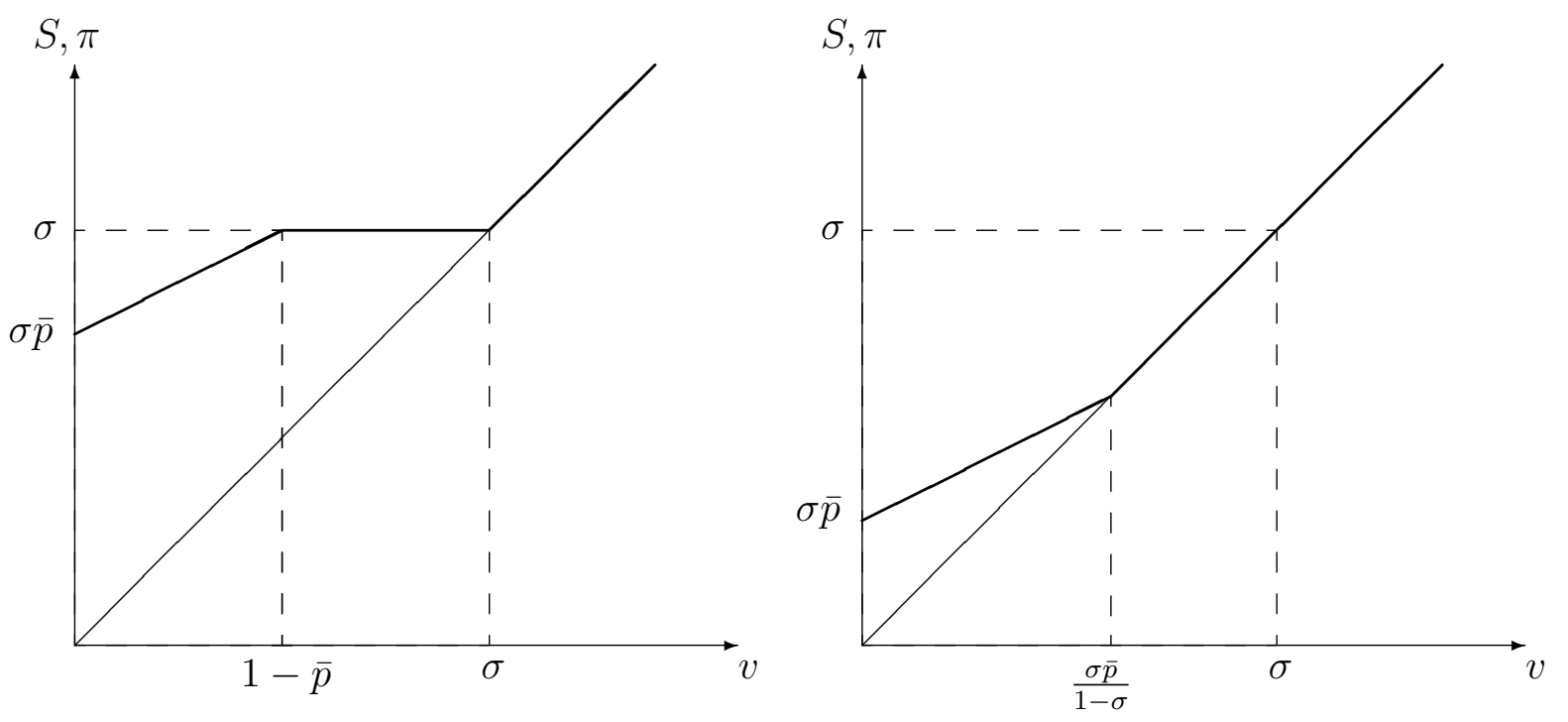

Figure 4: Profits with a reference price when (i) $\bar{p} \geq 1-\sigma$ and (ii) $\bar{p}<1-\sigma$.

Lemma 4 We can distinguish two cases: (i) If $\bar{p} \geq 1-\sigma$, the equilibrium price is

$$
p= \begin{cases}v & \text { if } v \geq \sigma \\ 1 & \text { if } 1-\bar{p} \leq v<\sigma \\ v+\bar{p} & v<1-\bar{p}\end{cases}
$$

(ii) If $\bar{p}<1-\sigma$ the price is

$$
p= \begin{cases}v & \text { if } v \geq \frac{\sigma \bar{p}}{1-\sigma} \\ v+\bar{p} & \text { if } v<\frac{\sigma \bar{p}}{1-\sigma} .\end{cases}
$$

Profits are $\pi=v$ when $p=v$ and the whole market is covered, and otherwise $\pi=\sigma p$.

Regarding the R\&D decision of firm $i$, we denote $\theta_{R P}^{*}(\sigma, \bar{p})$ the level of investment. Figure $4(i)$ and 4 (ii) illustrate the profits of the firm for different realizations of $v$ in each of the two scenarios mentioned in the lemma. For case (i), the optimal investment of the firm corresponds to the solution to

$$
\theta_{R P}^{*}(\sigma, \bar{p}) \in \arg \max _{\theta} \int_{0}^{1-\bar{p}} \sigma(v+\bar{p}) f(v, \theta) d v+\int_{1-\bar{p}}^{\sigma} \sigma f(v, \theta) d v+\int_{\sigma}^{1} v f(v, \theta) d v-\theta .
$$

In case (ii) the optimal investment is obtained as

$$
\theta_{R P}^{*}(\sigma, \bar{p}) \in \arg \max _{\theta} \int_{0}^{\frac{\sigma \bar{p}}{(1-\sigma)}} \sigma(v+\bar{p}) f(v, \theta) d v+\int_{\frac{\sigma \bar{p}}{(1-\sigma)}}^{1} v f(v, \theta) d v-\theta .
$$


From Lemma 4, a higher reference price leads to higher optimal prices (and profits) for low realizations of $v$, since $\bar{p}$ is only relevant for the captured doctors. In fact, the comparison of both cases shows that in case (i) profits for firm $i$ are larger for low realizations of $v$. These excessive returns for low values of $v$ reduce more the incentives to invest in case (i) in comparison with case (ii). Furthermore, this intuition can be generalized to any value of the reference price, so that the higher is $\bar{p}$ the lower is $\theta_{R P}^{*}$.

Proposition $3 \theta_{R P}^{*}(\sigma, \bar{p}) \leq \theta^{S}$ is decreasing in $\bar{p}$.

As expected, when $\bar{p}$ goes to 0, equation (3) converges to the planner's problem and the first best is thus achieved. Similarly, when $\bar{p}$ converges to 1, equation (2) converges to the benchmark monopoly case.

We have therefore shown that the reference price is an effective tool to align the incentives of the firm and the society. However, because the optimal investment level is only achieved when $\bar{p}=0$, no inefficiency is linked to the lack of insurance to patients. This extreme result is opposed to the characterization of the copayment scheme, for which efficiency can be obtained even with a modest level of subsidization $1-\alpha$. These two different results arise from the way each mechanism affects the demand elasticity of the captured doctors. While in the case of the reference price, decreases in $\bar{p}$ represent a constant shift in the price of the firm for low realizations of $v$, increases in the copayment decrease the slope of the profit profile.

\section{Competition}

We now turn to the study of the effects of the captured doctors in the context of competition. As we have shown, in the case of a single monopolist, the existence of captured doctors decreases the demand elasticity, specially for small innovations, and results in a reduction in the innovative effort of the firm. Under oligopoly, we will show that the result is in general unclear, since firms will also try to be the one with the leading improvement. It is also important to emphasize that in the environment we consider, the typical distortions associated to patent races characterized 
for example in Loury (1979) do not arise and, absent the captured doctors, the first best would be achieved with the decentralized choice of firms' effort.

In particular, suppose that starting from the situation where a good of quality 0 is produced competitively, two firms $i=1,2$ can obtain an improvement. Both firms share the same technology, which implies that the quality of their improvements originates from the same distribution $f(v, \theta)$, and incur the same cost of investment $\theta$.

The first best is easy to characterize. Given that all patients are identical and that the marginal cost of production is identical for all goods, if firm 1 and 2 obtain an improvement $v_{1}$ and $v_{2}$ respectively, the social optimum implies that only the good with the highest quality is produced and consumed by all agents. Therefore, the social welfare problem can be written as follows,

$$
\max _{\theta_{1}, \theta_{2}} \int_{0}^{1}\left\{\int_{0}^{v_{2}} v_{2} f\left(v_{1}, \theta_{1}\right) d v_{1}+\int_{v_{2}}^{1} v_{1} f\left(v_{1}, \theta_{1}\right) d v_{1}\right\} f\left(v_{2}, \theta_{2}\right) d v_{2}-\theta_{1}-\theta_{2}
$$

and the symmetric solution is denoted as $\theta_{1}^{s}=\theta_{2}^{s}=\theta^{s} .{ }^{10}$

In the competitive solution, absent the captured doctors, if firm 1 and 2 charge prices $p_{1}$ and $p_{2}$ respectively, doctors prefer good 1 to good 2 only if

$$
v_{1}-p_{1} \geq v_{2}-p_{2}
$$

Again, since all firms have a marginal cost of production of 0, standard arguments related to Bertrand competition lead to the outcome that if, for example, $v_{1}>v_{2}$, then $p_{1}=v_{1}-v_{2}$ and $p_{2}=0$. Profits would result in $\pi_{1}=v_{1}-v_{2}$ and $\pi_{2}=0$.

In the first stage, firm 1 and 2 choose the investment simultaneously. Firm 1 , given $\theta_{2}$, chooses the level of investment that maximizes,

$$
\max _{\theta_{1}} \int_{0}^{1}\left\{\int_{v_{2}}^{1}\left(v_{1}-v_{2}\right) f\left(v_{1}, \theta_{1}\right) d v_{1}\right\} f\left(v_{2}, \theta_{2}\right) d v_{2}-\theta_{1},
$$

which results in a reaction function $\theta_{1}\left(\theta_{2}\right)$. The equilibria of the game are denoted as $\left(\theta_{1}^{*}, \theta_{2}^{*}\right)$. The next lemma, shows that the investment chosen privately by firms coincide with the first best.

\footnotetext{
${ }^{10}$ Notice that by focussing on the symmetric solution we exclude scale economies in research that would lead to only one firm investing.
} 
Lemma 5 When $\sigma=0$, in the symmetric equilibrium of the game $\theta_{1}^{*}=\theta_{2}^{*}=\theta^{s}$.

As this lemma shows, competition between both firms does not generate any inefficiency in this environment. This result is due to the fact that each firm appropriates only of the surplus it generates. In other words, each firm implicitly internalizes in its research effort the negative marginal effect it has on the profits accrued by the competitor. Figure 5(i) makes this argument clear by comparing the social contribution of a firm with its private profits. This is the reason why, compared to the usual case of patent races where the winner-takes-all prevails, we do not obtain overinvestment. This feature of the equilibrium turns out to be convenient in order to study the effect of the captured doctors.

This equivalence result does in general not hold for a general value of $\sigma$. To see it, consider the opposite case, characterized by $\sigma=1$. There, in the competitive solution it would still be the case that the firm with the highest quality sells the good, prescribed by the captured doctors. The price is limited by the regulated ceiling, $p_{i}=1$. The other firm does not sell. Hence, the effort choice of firm 1, solves

$$
\max _{\theta_{1}} \int_{0}^{1} \int_{v_{2}}^{1} f\left(v_{1}, \theta_{1}\right) d v_{1} f\left(v_{2}, \theta_{2}\right) d v_{2}-\theta_{1}=\max _{\theta_{1}} \int_{0}^{1}\left(1-F\left(v_{2}, \theta_{1}\right)\right) f\left(v_{2}, \theta_{2}\right) d v_{2}-\theta_{1}
$$

The comparison with the first best case (and the situation with $\sigma=0$ ) is in general unclear. As Figure 5(ii) illustrates, with a larger $\sigma$ each firm has more incentives to reach an innovation larger than the competitor. However, provided that a firm achieves an innovation larger than the competitor, the marginal return from a larger improvement is lower than its social contribution. To illustrate these two forces we now discuss a simple example.

\subsection{An Example}

Let's assume that $f(v, \theta)$ is such that there are two possible realizations of $v, \underline{v}$ and $\bar{v}$. We parameterize these realizations as $\underline{v}=\frac{1}{2}-x$ and $\bar{v}=\frac{1}{2}+x$, where $0 \leq x \leq \frac{1}{2}$ is a measure of the risk of the innovative process. Given an investment level $\theta_{i}$, the probability of obtaining the high realization is $\theta_{i}^{\frac{1}{2}}$. 

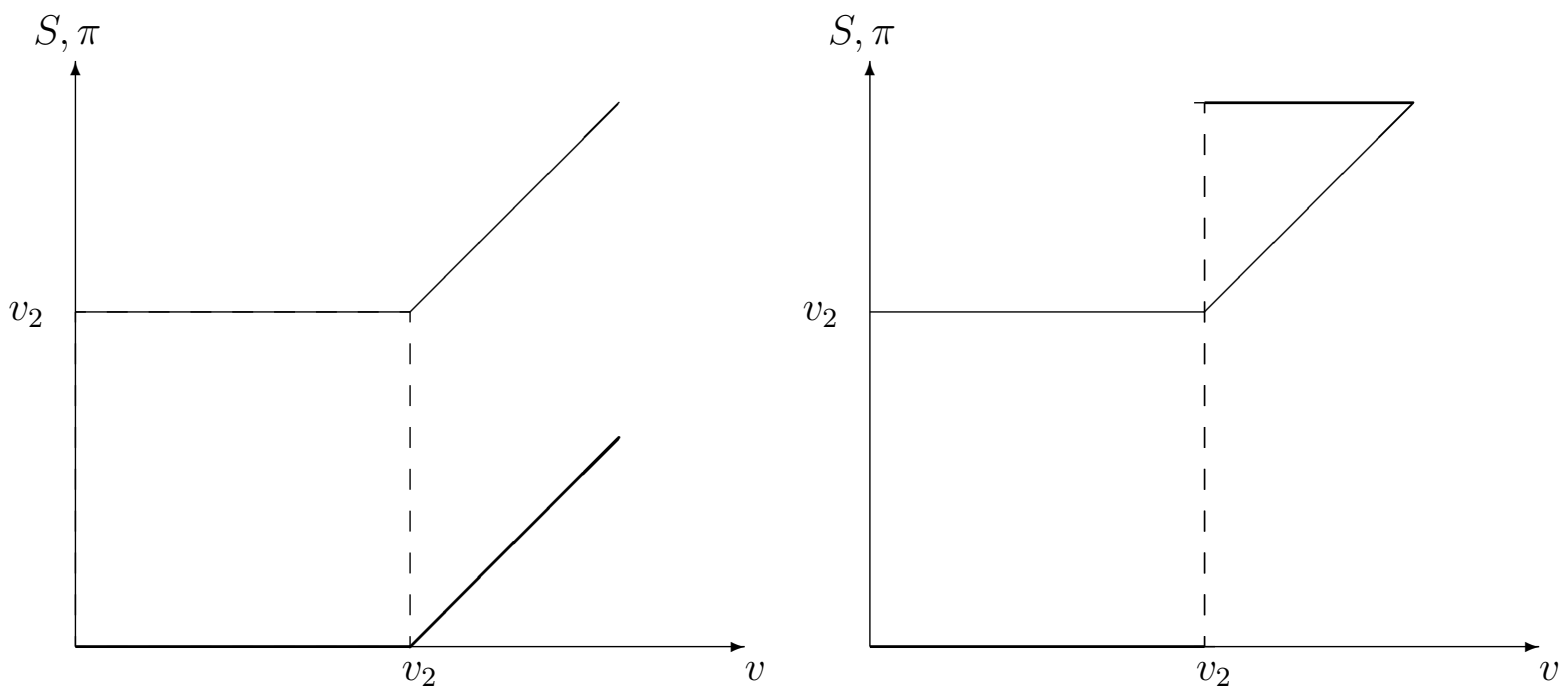

Figure 5: Profits of firm 1 and social welfare generated as a function of $v_{1}$ for a given value of $v_{2}$ when (i) $\sigma=0$ and when (ii) $\sigma=1$.

We first solve for the first best using the discrete analog of equation (4) as

$$
\max _{\theta_{1}, \theta_{2}} \theta_{1}^{\frac{1}{2}} \theta_{2}^{\frac{1}{2}} \bar{v}+\theta_{1}^{\frac{1}{2}}\left(1-\theta_{2}^{\frac{1}{2}}\right) \bar{v}+\left(1-\theta_{1}^{\frac{1}{2}}\right) \theta_{2}^{\frac{1}{2}} \bar{v}+\left(1-\theta_{1}^{\frac{1}{2}}\right)\left(1-\theta_{2}^{\frac{1}{2}}\right) \underline{v}-\theta_{1}-\theta_{2} .
$$

Hence, only when both firms obtain a low realization of $v$, the good with value $\underline{v}$ is consumed. The unique solution of this problem implies

$$
\theta_{1}^{s}=\theta_{2}^{s}=\theta^{s}=\left(\frac{\bar{v}-\underline{v}}{2+\bar{v}-\underline{v}}\right)^{2}=\left(\frac{x}{1+x}\right)^{2} .
$$

For $\sigma=0$ the maximization problem of firm 1 can be written as

$$
\max _{\theta_{1}} \theta_{1}^{\frac{1}{2}}\left(1-\theta_{2}^{\frac{1}{2}}\right)(\bar{v}-\underline{v})-\theta_{1} .
$$

As stated in Lemma 5, the Nash Equilibrium of the game also implements the first best.

However, when $\sigma=1$, the payoffs of each of the firms are as follows. When both firms obtain the same improvement, both charge a price equal to 1 and share profits equally, so that each obtains $\frac{1}{2} \cdot{ }^{11}$ When a firm obtains an improvement $\bar{v}$ and the competitor $\underline{v}$ profits are 1 .

\footnotetext{
${ }^{11}$ Given that doctors are not sensitive to price reductions, other equilibria can arise. However, for the purposes of this example other symmetric equilibria have identical implications.
} 
Hence, the problem of the firm can be written as

$$
\max _{\theta_{1}} \theta_{1}^{\frac{1}{2}} \theta_{2}^{\frac{1}{2}} \frac{1}{2}+\theta_{1}^{\frac{1}{2}}\left(1-\theta_{2}^{\frac{1}{2}}\right)+\left(1-\theta_{1}^{\frac{1}{2}}\right)\left(1-\theta_{2}^{\frac{1}{2}}\right) \frac{1}{2}-\theta_{1}
$$

and in equilibrium $\theta_{1}^{*}=\theta_{2}^{*}=\frac{1}{16}$.

Comparing the market equilibrium with the first best we observe that when $x<\frac{1}{3}$ the competitive equilibrium yields excessive innovation while the opposite is true for $x>\frac{1}{3}$. As discussed above, the intuition for the result is the following. When $x$ is very small, the productivity of effort is small. However, the profits from obtaining the high innovation are above the social welfare generated, leading to overinvestment. An opposing force arises from the lack of sensitivity of profits to increases in the value of the high realization. For this reason, when $x$ is large the first best would require more investment, yet the equilibrium investment is unchanged because the private return from innovation is independent of $x$.

\section{Concluding Remarks}

This paper presents a stylized model of innovation that emphasizes the distortions induced on innovation by the different layers of incentives in the relationship between patients and pharmaceutical firms. We have exemplified these distortions in the differentiated behavior of some doctors, depending on whether they perform an efficient cost-benefit analysis of each product or if instead they internalize the preferences of their patients. Although this is a rough description of the doctors' conduct, it is consistent with empirical evidence obtained using prescription data.

A more structural model should explicitly consider the behavior of doctors and in particular, how they are affected by the level of advertising of pharmaceutical companies. As it has been documented, doctors are not necessarily aware of all products available, their prices and qualities and they might be subject to manipulation by these firms. Competition between pharmaceutical companies to capture doctors as opposed to competition to obtain better products might be an interesting avenue for further research. 
Nevertheless, the results of the paper essentially rely on the lower than efficient price elasticity of some doctors. Regardless of whether this low elasticity originates from the doctor capture by patients or pharmaceutical firms, or it is just due to the lack of information of these doctors, the general message of this paper remain unchanged. The level of research that firms undertake depends not only on the total rents from innovation, but also - once we take into account that the level of innovation is endogenous - on the rewards that the firm obtains for any size of innovation it might achieve. In this sector, low price elasticity entails a bias towards small innovations.

Finally, this paper has policy implications for the debate on the effects of regulation of pharmaceutical expenditure. Pharmaceutical companies often claim that instruments aimed to reduce the agency problem between doctors and insurers might reduce the incentives to innovate. Our results suggest that these instruments might indeed reduce the reward from some innovations, the social value of which is small, yet they induce higher incentives to obtain larger innovations. 


\section{A Heterogeneity in the Doctors' Capture}

We now extend the benchmark model to allow for heterogeneity in the degree of doctor's capture. As we will see, this heterogeneity allows us to dispense with the assumption that the price is constrained to be below 1 , yet the results will remain essentially unchanged. ${ }^{12}$ We still assume that the proportion $1-\sigma$ of non-captured doctors that perform the cost-benefit analysis is homogeneous. However, captured doctors are heterogeneous in a parameter $s$ that is distributed uniformly between 0 and 1 , and their utility corresponds to

$$
U_{C}(v)=v+s-p
$$

Hence, doctors do not share the same preferences than their patients, and instead they overestimate the effect that the new product has on them. This additional value $s$ can be rationalized, for example, as the increase in the quality perception derived from advertising. The resulting demand function for firm $i$ can be described as

$$
D(p)= \begin{cases}1 & \text { if } p \leq v \\ \sigma(v+1-p) & \text { if } v<p \leq 1+v \\ 0 & \text { otherwise }\end{cases}
$$

As before, firm $i$ might choose between selling to all consumers or only to the non-sensitive part of the demand. While for the earlier, the optimal price still corresponds to $p=v$, for the latter, the optimal price maximizes

$$
\max _{p} \sigma(1+v-p) p
$$

which results in $p^{*}=\frac{1+v}{2}$. Compared to the benchmark model, the relevant price and the quantity sold when firm $i$ targets the inelastic part of the demand are strictly increasing in $v$. Comparing profits in both cases, we obtain the following result.

Lemma 6 The optimal price for firm $i$ corresponds to

$$
p^{*}= \begin{cases}v & \text { if } v \geq v^{*}(\sigma), \\ \frac{1+v}{2} & \text { otherwise }\end{cases}
$$

\footnotetext{
${ }^{12}$ Since the need for this price limit is due to the fact that captured doctors induce a completely inelastic demand, we could alternatively assume that doctors have a positive yet lower than optimal price elasticity.
} 
where $v^{*}(\sigma)=\frac{2}{\sigma}(1-\sqrt{1-\sigma})-1$ is increasing in $\sigma$. Profits can be written as

$$
\pi(v)= \begin{cases}v & \text { if } v \geq v^{*}(\sigma), \\ \sigma\left(\frac{1+v}{2}\right)^{2} & \text { otherwise }\end{cases}
$$

with $\pi^{\prime}(v)<1$ when $v<v^{*}(\sigma)$.

Notice that similarly to the benchmark model, for low values of $v$, profits for firm $i$ are higher than the surplus generated. Moreover, profits in this range rise with increases in $v$ less than the social welfare generated. The next proposition shows that in any such case the firm will invest less than what is socially optimal.

Proposition 4 For any $\pi(v)$ increasing in $v$ such that $\pi^{\prime}(v) \leq 1, \theta^{*} \leq \theta^{S}$, with strict inequality if there exists a range of $v$ for which $\pi^{\prime}(v)<1$ and $f(v, \theta)>0$ for all $\theta$.

This proposition also allows us to contribute to the classical debate on how to provide incentives to innovate. Most papers have mainly focused on the trade-off between assigning monopoly power to innovators in order to internalize the returns from their research and the minimization of the resulting dead-weight loss. While often the size of the innovation that firms achieve is assumed to be exogenous, here it is the object of our study. As a result, we show that although firms might receive a reward from their innovation higher than their social contribution, underinvestment might occur if the increase in this reward when higher effort in research is exerted is lower than socially optimal.

Finally, notice that we have undertaken this analysis in a context where no dead-weight loss from monopoly power arises in order to emphasize our results. However, the possibility of this dead-weight loss is not likely to change the results, since it would correspond to an additional force reducing the incentives to innovate. 


\section{B Proofs}

\section{B.1 Preliminary Result and Notation}

We will make repeated use of the following very well-known result, which we state as a lemma: if $X \geq_{s t} Y$ then for all increasing functions $\psi, E[\psi(X)] \geq E[\psi(Y)]$.

Lemma 7 Let $X$ and $Y$ be real-valued random variables with cumulative distribution functions $F$ and $G$ respectively, such that $F(z) \leq G(z)$ for all $z \in \mathbf{R}$. For all bounded real-valued increasing functions $\psi: \mathbf{R} \rightarrow \mathbf{R}$,

$$
\int_{\mathbf{R}} \psi(z) \mathrm{d} F(z) \geq \int_{\mathbf{R}} \psi(z) \mathrm{d} G(z)
$$

\section{B.2 Proofs of the Results}

Proof of Lemma 1: Immediate from the arguments in the main text.

Proof of Proposition 1: The firm's profit function is

$$
\pi(\sigma, \theta)=\int_{0}^{\sigma} \sigma f(v, \theta) d v+\int_{\sigma}^{1} v f(v, \theta) d v-\theta .
$$

Using the results of Milgron and Shannon (1994) it is enough to show, that $\pi(\sigma, \theta)$ has decreasing differences. Since

$$
\frac{\partial \pi(\sigma, \theta)}{\partial \sigma}=\int_{0}^{\sigma} f(v, \theta) d v=F(\sigma, \theta)
$$

and from our assumptions on $F, \frac{\partial \pi(\sigma, \theta)}{\partial \sigma}$ is decreasing in $\theta$, which concludes the proof.

Proof of Lemma 2: Immediate from the arguments in the main text.

Proof of Lemma 3: Immediate from the arguments in the main text.

Proof of Proposition 2: Part (ii) is immediate from the fact that when $\alpha>\sigma$ the problem of the firm coincides with the social planner's problem.

For part (i) notice that

$$
\pi(\sigma, \alpha, \theta)-\pi(\sigma, 0, \theta)=\int_{0}^{\alpha} \sigma\left[\frac{v}{\alpha}-1\right] f(v, \theta) d v
$$


and using Lemma 7, $\pi(\sigma, \alpha, \theta)-\pi(\sigma, 0, \theta)$ is increasing in $\theta$ given that

$$
H(v)= \begin{cases}0 & \text { if } v \geq \alpha \\ \sigma\left[\frac{v}{\alpha}-1\right] & \text { if } v<\alpha .\end{cases}
$$

is weakly increasing in $v$. The results of Milgron and Shannon (1994) and the fact that $\pi(\sigma, \alpha, \theta)-\pi(\sigma, 0, \theta)$ is increasing in $\theta$ imply that $\theta^{*}(\sigma) \leq \theta_{C O}^{*}(\sigma, \alpha) \leq \theta^{S}$.

Proof of LEMma 4: Immediate from the arguments in the main text.

Proof of Proposition 3: Two cases need to be analyzed. When (i) $\bar{p}^{\prime}>\bar{p} \geq 1-\sigma$, then

$$
\pi(\sigma, \bar{p}, \theta)-\pi\left(\sigma, \bar{p}^{\prime}, \theta\right)=\int_{0}^{1-\bar{p}^{\prime}} \sigma\left(\bar{p}-\bar{p}^{\prime}\right) f(v, \theta) d v+\int_{1-\bar{p}^{\prime}}^{1-\bar{p}} \sigma(v+\bar{p}-1) f(v, \theta) d v
$$

Notice that, by Lemma $7, \pi(\sigma, \bar{p}, \theta)-\pi\left(\sigma, \bar{p}^{\prime}, \theta\right)$ is increasing in $\theta$ given that the function

$$
H(v)= \begin{cases}0 & v>1-\bar{p} \\ \sigma(v+\bar{p}-1) & \text { if } 1-\bar{p}^{\prime} \leq v<1-\bar{p}, \\ \sigma(\bar{p}-\bar{p}) & \text { if } v<1-\bar{p}^{\prime}\end{cases}
$$

is weakly increasing in $v$.

Similarly, if $\bar{p}^{\prime}<\bar{p}<1-\sigma$.

$$
\pi(\sigma, \bar{p}, \theta)-\pi\left(\sigma, \bar{p}^{\prime}, \theta\right)=\int_{0}^{\frac{\sigma \bar{p}}{1-\sigma}} \sigma\left(\bar{p}-\bar{p}^{\prime}\right) f(v, \theta) d v+\int_{\frac{\sigma \bar{p}}{1-\sigma}}^{\frac{\sigma \bar{p}^{\prime}}{1-\sigma}}\left(v-\sigma\left(v+\bar{p}^{\prime}\right)\right) f(v, \theta) d v
$$

Where again, by Lemma $7 \pi(\sigma, \bar{p}, \theta)-\pi\left(\sigma, \bar{p}^{\prime}, \theta\right)$ is increasing in $\theta$ due to the fact that the function

$$
H(v)= \begin{cases}0 & v>\frac{\sigma \bar{p}^{\prime}}{1-\sigma} \\ v-\sigma\left(v+\bar{p}^{\prime}\right) & \text { if } \frac{\sigma \bar{p}}{1-\sigma} \leq v<\frac{\sigma \bar{p}^{\prime}}{1-\sigma}, \\ \sigma\left(\bar{p}-\bar{p}^{\prime}\right) & \text { if } v<\frac{\sigma \bar{p}}{1-\sigma} .\end{cases}
$$

is weakly increasing. The results of Milgron and Shannon (1994) and the fact that $\pi(\sigma, \bar{p}, \theta)-$ $\pi\left(\sigma, \bar{p}^{\prime}, \theta\right)$ is increasing in $\theta$ imply that $\theta_{R P}^{*}(\sigma, \bar{p})$ is decreasing in $\bar{p}$. Finally, given that $\theta_{R P}^{*}(\sigma, 1)=$ $\theta^{S}$ since when $\bar{p}=1$ the firm's and social planner's problem coincide, we can conclude that $\theta_{R P}^{*}(\sigma, \bar{p}) \leq \theta^{S}$ for all $\bar{p}$.

Proof of Lemma 5: The social planer problem

$$
\max _{\theta_{1}, \theta_{2}} \int_{0}^{1}\left\{\int_{0}^{v_{2}} v_{2} f\left(v_{1}, \theta_{1}\right) d v_{1}+\int_{v_{2}}^{1} v_{1} f\left(v_{1}, \theta_{1}\right) d v_{1}\right\} f\left(v_{2}, \theta_{2}\right) d v_{2}-\theta_{1}-\theta_{2}
$$


By adding and subtracting $\int_{v_{2}}^{1} v_{2} f\left(v_{1}, \theta_{1}\right) d v_{1}$, we obtain

$$
\max _{\theta_{1}, \theta_{2}} \int_{0}^{1}\left\{v_{2}+\int_{v_{2}}^{1}\left(v_{1}-v_{2}\right) f\left(v_{1}, \theta_{1}\right) d v_{1}\right\} f\left(v_{2}, \theta_{2}\right) d v_{2}-\theta_{1}-\theta_{2}
$$

If we compare this problem with the problem of the firm,

$$
\max _{\theta_{1}} \int_{0}^{1}\left\{\int_{v_{2}}^{1}\left(v_{1}-v_{2}\right) f\left(v_{1}, \theta_{1}\right) d v_{1}\right\} f\left(v_{2}, \theta_{2}\right) d v_{2}-\theta_{1} .
$$

We realize that the first order conditions over $\theta_{1}$ must coincide in both problems for a given $\theta_{2}$. We can do the same with respect to $\theta_{2}$, obtaining the same conclusion. This concludes the proof.

Proof of Lemma 6: Immediate from the arguments in the text.

Proof of Proposition 4:

The optimal investment of firm $i$ satisfies in this case

$$
\theta^{*} \in \arg \max _{\theta} \int_{0}^{1} \pi(v) f(v, \theta) d v-\theta .
$$

So, let's consider the following problem. Given a parameter $\gamma \in[0,1]$, denote as $H$ the following function

$$
H(\theta, \gamma) \equiv \int_{0}^{1}(\gamma \pi(v)+(1-\gamma) v) f(v, \theta) d v-\theta
$$

and define $\theta(\gamma)$ as

$$
\theta(\gamma) \in \arg \max _{\theta} H(\theta, \gamma)
$$

Notice that $\theta(\gamma)$ is decreasing in $\gamma$ if $H(\theta, \gamma)$ has decreasing differences in $\theta$ and $\gamma$. Taking the derivative of $H$ with respect to $\gamma$ and integrating by parts, we obtain

$$
\frac{\partial H}{\partial \gamma}=\pi(1)-\int_{0}^{1}\left(\pi^{\prime}(v)-1\right) F(v, \theta) d v
$$

and differentiating with respect to $\theta$,

$$
\frac{\partial H}{\partial \gamma \partial \theta}=-\int_{0}^{1}\left(\pi^{\prime}(v)-1\right) \frac{\partial F}{\partial \theta}(v, \theta) d v
$$

which is negative if $\pi^{\prime}(v)<1$. 


\section{References}

Bulow, Jeremy, "The Gaming of Pharmaceutical Patents," in Adam B. Jaffe, Scott Stern and Josh Lerner, eds., Innovation Policy and the Economy, Volume 4, NBER Books, MIT Press, 2004 .

Caves, R.E, M. D. Whinston and M. A. Hurwitz, "Patent Expiration, Entry and Competition in the U.S. Pharmaceutical Industry," Brookings Papers on Economic Activity, Microeconomics, 1991, pp. 1-48.

Danzon, P.M. And L.W. ChaO, "Does Regulation Drive Out Competition in the Pharmaceutical Markets?" Journal of Law and Economics, 2000, XLIII, pp. 311-357.

Frank, Richard G. and David S. Salkever, "Pricing, Patent Loss and the Market For Pharmaceuticals," Southern Economic Journal, 1992, 59, pp. 165-179.

Gilbert, Richard And CARl Shapiro, "Optimal Patent Length and Breadth," RAND Journal of Economics, 1990, 21(1), pp. 106-112.

Hellerstein, Judith K., "The Importance of the Physician in the Generic versus TradeName Prescription Decision," RAND Journal of Economics, 1998, 29(1), pp. 108-136.

Hollis, Aidan, "An Efficient Reward System for Pharmaceutical Innovation," 2005, mimeo.

Klemperer, PAul, "How Broad Should the Scope of Patent Protection Be?" RAND Journal of Economics, 1990, 21(1), pp. 113-130.

Lexchin, Joel, "Intellectual Property Rights and the Canadian Pharmaceutical Marketplace: Where Do we Go From Here?" 2003, available at http://www.policyalternatives.ca/publications/ipr.pdf.

Loury, GLenn, "Market Structure and Innovation," The Quarterly Journal of Economics, 1979, 93, pp. 395-410. 
Love, JAmes, "Evidence Regarding Research and Development Investment in Innovative and Non-Innovative Medicines," 2003, available at http://www.cptech.org/ip/health/md/evidenceregardingrnd.pdf.

Milgron, Paul And Chris Shannon, "Monotone Comparative Statics," Econometrica, 1994, 62(1), pp. 157-180.

NIHCM, "Changing Patterns of Pharmaceutical Innovation," May 2002.

Nordhaus, William, Invention, Growth and Welfare, MIT Press, 1969.

Scherer, F.M., "Pricing, Profits, and Technological Progress in the Pharmaceutical Industry," Journal of Economic Perspectives, 1993, 7(3), pp. 97-115.

Scotchmer, Suzanne, "On the Optimality of the Patent Renewal System," RAND Journal of Economics, 1999, 30, pp. 181-196. 